\title{
In Situ Synchrotron X-ray Diffraction Studies of Single-walled Carbon Nanotubes for Electric Double-layer Capacitors
}

\author{
Yukihiro Yoshida, Masato Tsutsui, Ayar Al-zubaidi, Yosuke Ishii and Shinji Kawasaki* \\ Nagoya Institute of Technology, Gokiso, Showa, Nagoya, Aichi 4668555, Japan.
}

\begin{abstract}
In situ synchrotron X-ray diffraction experiments of SWCNT (single-walled carbon nanotube) electrode in alkali halide aqueous electrolyte at several applied potentials were performed, and the change in the diffraction pattern of SWCNTs was observed. It was found that the position of the 100 diffraction peak does not change with applied potential while the peak intensity decreases with anion adsorption. It was concluded that the space inside the tube would be the important ion adsorption site for the well-grown SWCNT bundles.
\end{abstract}

Key words: Carbon nanotubes, synchrotron X-ray, EDLC.

\section{Introduction}

Owing to their high power properties, EDLCs (electric double-layer capacitors) have attracted much interest. EDLCs store electric energy just by physical adsorption of electrolyte ions on the surface of electrodes. Activated carbons are usually used for EDLC electrodes because they have high specific surface area. However, the energy density of EDLCs is much lower than that of secondary batteries such as $\mathrm{Li}$ ion battery. Therefore, there is a strong demand to develop new electrode materials to improve the energy density of EDLC. SWCNTs (Single-walled carbon nanotubes) have been expected as a good electrode material for EDLC because the theoretical specific surface area of SWCNTs is very large $(2,630$ $\mathrm{m}^{2} / \mathrm{g}$ ) [1-4]. However, the reported EDLC capacitance values of SWCNTs are not so high compared to the values of the typical EDLC electrodes (e.g. activated carbon). One of the reasons why the expected high value could not be obtained is that SWCNTs exist in the form of aggregations (bundle structure), in which

\footnotetext{
*Corresponding author: Shinji Kawasaki, professor, research field: carbon chemistry.
}

individual SWCNTs are gathered due to van der Waals interaction. Due to this aggregation form, the actual specific surface area of the aggregated SWCNTs is much lower than that of an isolated single SWCNT. On the other hand, it is interestingly also known that the EDLC capacitance of the bundled SWCNTs per unit surface area is much higher than that of activated carbons [1]. However, the ion adsorption mechanism of the bundled SWCNTs has not been understood well. In order to elucidate the mechanism, the authors performed in situ synchrotron XRD measurements of the bundled SWCNTs during adsorption of electrolyte ions.

\section{Experiments}

In the present study, the authors used high quality SWCNT sample (Meijo Nano Carbon Co Ltd., So Type). The quality and the purity of the SWCNT sample were characterized by TEM, XRD, and Raman measurements [5-7]. Ion adsorption process, that is the main topic in the present study, was investigated by in situ synchrotron XRD measurements of SWCNT electrode under applied potential. The XRD measurements were performed on a synchrotron 
radiation beam line $\mathrm{BL}-18 \mathrm{C}$ at the photon factory in High-Energy Accelerator Research Organization, Tsukuba, Japan. The beam was monochromatized by Si (111) double monochromators and collimated by a pinhole collimator of $100 \mathrm{~mm}$ in diameter. The incident X-ray wavelength was set to $\lambda=0.0613 \mathrm{~nm}$. For the in situ XRD measurements, the authors fabricated three electrode configuration cell that consists of SWCNT working, Pt counter, and $\mathrm{Ag} / \mathrm{AgCl}$ reference electrode. Several kinds of alkali halide aqueous electrolytes were used.

\section{Results and Discussions}

The Raman spectrum of the SWCNT sample (Fig. 1(a)) shows that the sample had high crystallinity and few defects because the intensity of the D-band at approximately $1,330 \mathrm{~cm}^{-1}$ was significantly weak. In addition, the RBM (radial breathing mode) peaks shown in the inset of Fig. 1(a) show small diameter distribution. Fig. 1(b) shows the observed and simulated XRD patterns of the bundled SWCNTs having 2-dimensional hexagonal structure [8]. As shown in Fig. 1(b), the simulated pattern reproduced the observed pattern very well. The mean tube

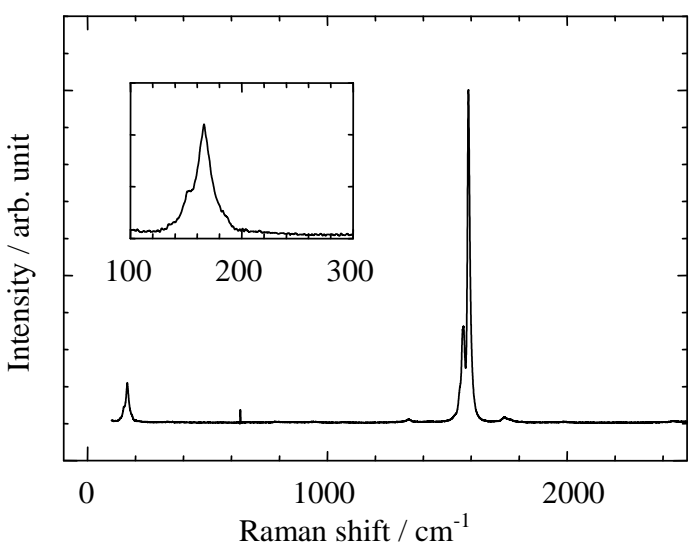

(a) diameter of $1.47 \mathrm{~nm}$ and the tube-center distance of $1.79 \mathrm{~nm}$ were used for the simulation. Therefore, the distance between surfaces of the nearest neighbor tubes is evaluated to be $0.31 \mathrm{~nm}$ which is in good agreement with the van der Waals radius of carbon atom.

In a previous study [7], the authors revealed that the cyclic voltammogram of SWCNT samples of high crystallinity and narrow diameter distribution had the shape of a dumbbell rather than either the typical rectangular shape obtained for other porous materials, or the butterfly shape previously reported for SWCNTs [3]. Fig. 2 shows that the observed cyclic voltammogram for the SWCNTs used in the present study is a dumbbell shape. The cyclic voltammogram experiment also confirmed that the SWCNTs used in the present study have high crystallinity.

Fig. 3(a) shows the change in XRD pattern of SWCNT electrode with increasing potential. As shown in Fig. 3(a), a very strong background noise related to the halo diffraction of electrolyte in the cell overlapped to XRD pattern of SWCNTs, obstructing the observation of higher order diffractions of SWCNTs. Therefore, the authors focused on the

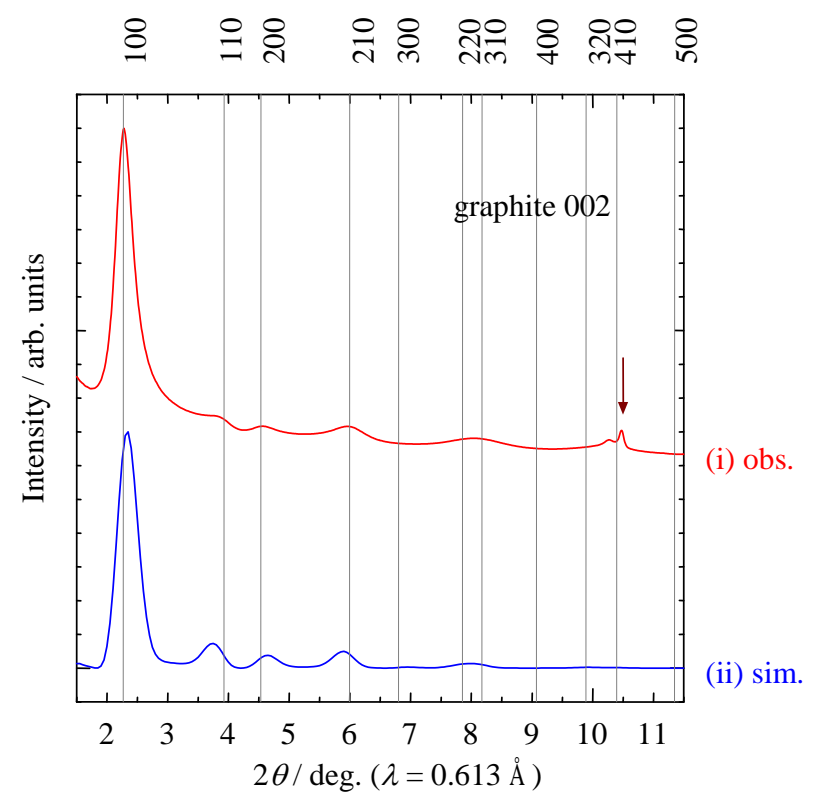

(b)

Fig. 1 (a) Raman spectrum; (b) Observed (i) and simulated (ii) X-ray diffraction patterns of SWCNTs. 


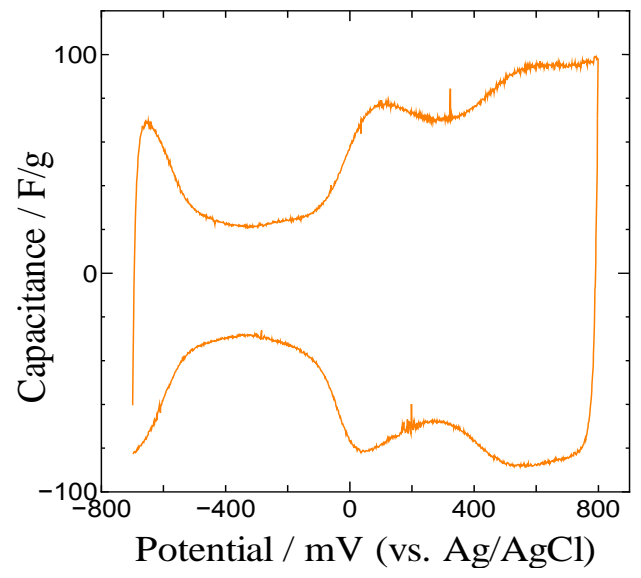

Fig. 2 Observed cyclic voltammogram of SWCNTs at potential scan rate of $10 \mathrm{mV} / \mathrm{s}$.

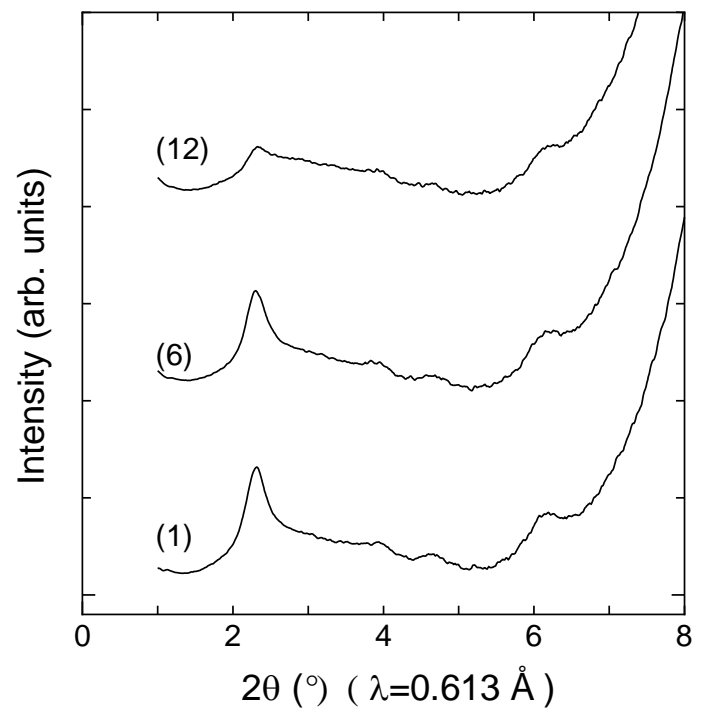

(a)

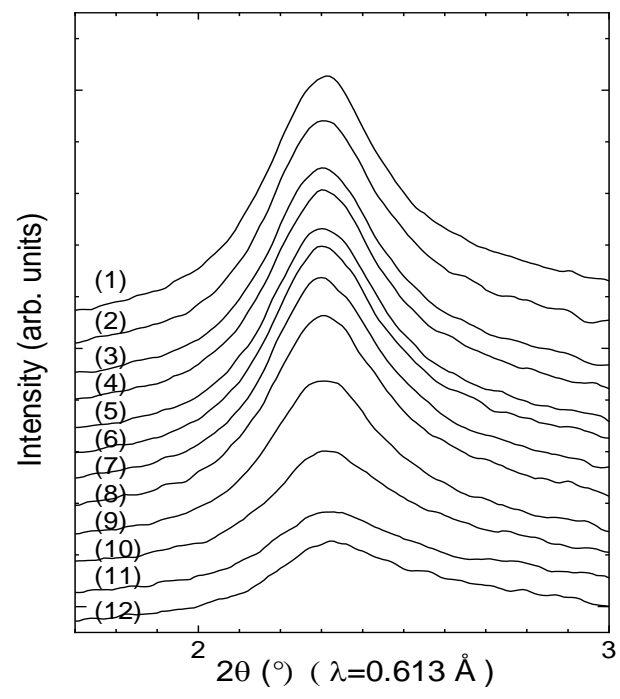

(b)

Fig. 3 (a) Observed XRD patterns of SWCNTs under applied potential; (b) Change in 100 diffraction peak profile as a function of applied potential $(1:-700 \mathrm{mV} ; 2:-550 \mathrm{mV}, 3:-400 \mathrm{mV} ; 4:-250 \mathrm{mV} ; 5:-100 \mathrm{mV} ; 6:+50 \mathrm{mV} ; 7:+200 \mathrm{mV} ; 8:+350 \mathrm{mV}$; 9: +500 mV; 10: +650 mV; 11: +800 mV; 12: +100 mV).

change in 100 diffraction peak (Fig. 3(b)). It was found that 100 peak position does not change with ion adsorption. On the other hand, the peak intensity was observed to decrease from $0 \mathrm{~V}$ with increasing potential (Fig. 4). According to Fig. 2, the potential range from 0 to $+800 \mathrm{mV}$ (Fig. 3(b)) corresponds to anion adsorption. To understand the change of the 100 diffraction with increasing potential, the authors have to understand the ion adsorption mechanism of the bundled SWCNTs. The probable ion adsorption sites of the bundled SWCNTs are: i) bundle outside surface; ii) the space inside the tube; iii) intertube sites. If ions are intercalated in an intertube site, the tube center-to-center distance should be enlarged. However, since the peak shift was not observed, the possibility of adsorption/intercalation in the intertube sites can be dismissed. Furthermore, the decrease of the peak intensity can be explained by ion adsorption inside the hollow cores of SWCNTs, because it is known that the 100 diffraction of SWCNTs loses its intensity by encapsulation other elements inside the tubes $[9,10]$. It should also be noted that the intensity decrease was more remarkable for the relatively heavier anions investigated in the present study. 


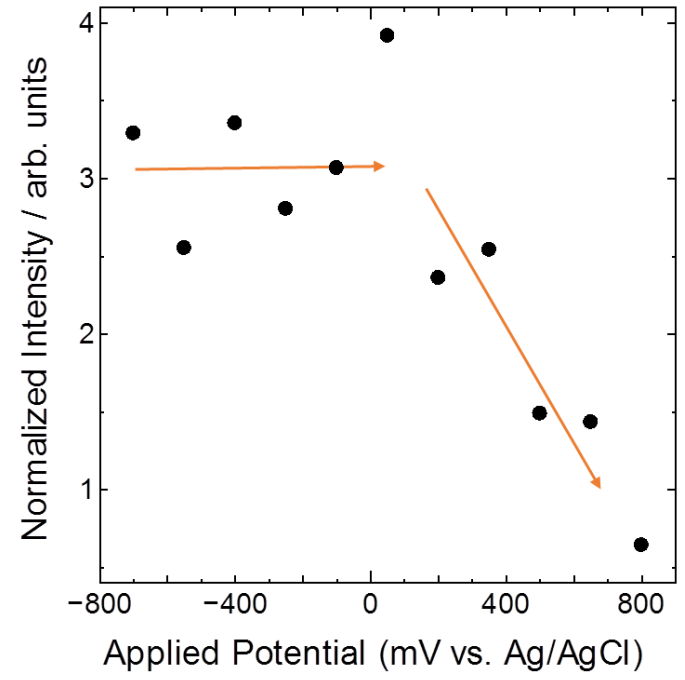

Fig. 4 Intensity of 100 diffraction peak as a function of applied potential.

The radius $R_{\mathrm{i}}$ of the intertube space shown in Fig. 5 (b) is expressed by the Eq. (1):

$$
R_{i}=0.15 \times R_{t}+0.19
$$

where, $R_{t}$ corresponds to the tube radius. In the present experiment, the tube radius $R_{\mathrm{t}}$ is $0.74 \mathrm{~nm}, R_{\mathrm{i}}$ is calculated to be $0.301 \mathrm{~nm}$ which is too small for solvated ions to be intercalated. On the other hand, the intensity of the 100 diffraction peak changed with applied potential. The intensity change indicates that ions are adsorbed inside the tubes, which shows that the inside of the tube is the important adsorption site. Eq. (2) expresses the surface ratio of bundle surface area $S_{\text {out }}$ to the tube inner surface area $S_{\text {in }}$ as a function of number $(N)$ of tubes in a bundle.

$$
S_{\text {out }} / S_{\text {in }}=\left(\frac{2 \sqrt{3}}{\pi N}\right)^{\frac{1}{2}}\left(\frac{2 R_{t}+2 r_{v d w}}{2 R_{t}-2 r_{v d w}}\right)
$$

where, $r_{\mathrm{vdw}}$ is a van der Waals radius of carbon atom. According to Eq. (2), the inside of the tube becomes the dominant surface with increasing bundle size. The bundle size of the present SWCNT sample was several tens of nanometers, which was estimated by the diffraction line width. Assuming the bundle size is 50 $\mathrm{nm}$, the tube inside surface area is about 5 times greater than the bundle outside surface area (Fig. 5(c)). Therefore, the main adsorption site of the bundled SWCNTs used in the present study would be tube inner surface site.

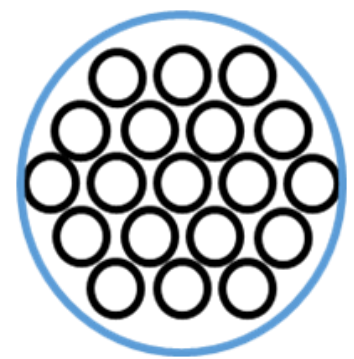

(a)

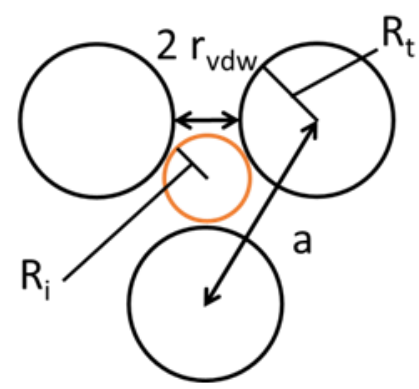

(b)

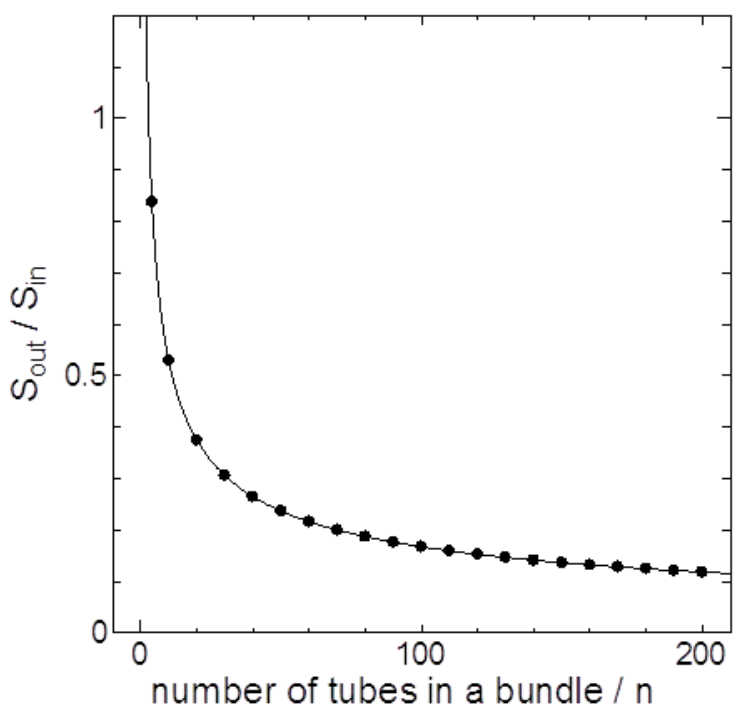

(c)

Fig. 5 (a) Schematic picture of SWCNT bundles; (b) Intertube space in a SWCNT triangular lattice; (c) Surface ratio of bundle outside surface area, $S_{\text {out }}$ to the tube inside surface area, $S_{\text {in }}$ as a function of number of tubes in a bundle. 


\section{Conclusions}

In situ synchrotron X-ray diffraction experiments of SWCNT electrode in alkali halide aqueous electrolyte solutions were performed at several applied potentials, and the change in the diffraction pattern of SWCNTs was observed. It was found that the position of the 100 diffraction peak does not change with applied potential, while the peak intensity decreases with anion adsorption. It was concluded that the inside of the tube would be the important ion adsorption site for the well grown SWCNT bundles.

\section{Acknowledgments}

The authors thank to Yashima Environment Technology Foundation, Toukai Foundation for Technology, TEPCO Memorial Foundation and JSPS KAKENHI Grant number 26410238 for their financial support to the present study.

\section{References}

[1] Shiraishi, S., Kurihara, H., Okabe, K., Hulicova, D., and Oya, A. 2002. "Electric Double Layer Capacitance of Highly Pure Single-walled Carbon Nanotubes." Electrochem. Commun 4: 593-8.

[2] Yamada, Y., Kimizuka, O., Machida, K., Suematsu, S., Tamamitsu, K., Saeki, S., Yamada, Y., Yoshizawa, N., et al.. 2010. "Hole-opening of Carbon Nanotubes and Their Capacitor Performance.” Energy Fuels 24: 3373-7.

[3] Hiraoka, T., Izadi-Najafabadi, A., Yamada, T., Futaba, D., Yasuda, S., Tanaike, O., Hatori, H., Yumura, M., et al..
2010. "Hata, K. Compact and Light Supercapacitor Electrodes from a Surface-Only Solid by Opened Carbon Nanotubes with 2,200 $\mathrm{m}^{2} \mathrm{~g}^{-1}$ Surface Area."Adv. Funct. Mater. 20: 422-8.

[4] Frackowiak, E., Lota, G., and Pernak, J. 2005. "Room-temperature Phosphonium Ionic Liquids for Supercapacitor Application." Appl. Phys. Lett. 86 (164104): 1-3.

[5] Al-zubaidi, A., Ishii, Y., Yamada, S., Matsushita, T., and Kawasaki, S. 2013. "Spectroscopic Evidence for the Origin of the Dumbbell Cyclic Voltammogram of Single-walled Carbon Nanotubes." Phys. Chem. Chem. Phys. 15: 20672-8.

[6] Al-zubaidi, A., Inoue, T., Matsushita, T., Ishii, Y., and Kawasaki, S. 2012. "Ion Adsorption on the Inner Surface of Single-walled Carbon Nanotubes Used as Electrodes for Electric Double-layer Capacitors." Phys. Chem. Chem. Phys. 14: 16055-61.

[7] Al-zubaidi, A., Inoue, T., Matsushita, T., Ishii, Y., Hashimoto, T., and Kawasaki, S. 2012. "Cyclic Voltammogram Profile of Single-Walled Carbon Nanotube Electric Double-Layer Capacitor Electrode Reveals Dumbbell Shape." J. Phys. Chem. C. 116: 7681-6.

[8] Thess, A., Lee, R., Nikolaev, P., Dai, H., Petit, P., Robert, J., Xu, C., Lee, Y., et al.. 1996. "Crystalline Ropes of Metallic Carbon Nanotubes.” Science 273: 483-7.

[9] Kawasaki, S., Iwai, Y., and Hirose, M. 2009. "Electrochemical Lithium Ion Storage Properties of Single-Walled Carbon Nanotubes Containing Organic Molecules." Carbon 47: 1081-6.

[10] Kawasaki, S., Iwai, Y., and Hirose, M. 2009. "Electrochemical Lithium Ion Storage Property of $\mathrm{C}_{60}$ Encapsulated Single-Walled Carbon Nanotubes." Mater. Res. Bull. 44: 415-7. 